\title{
AVALIAÇÃO DA ADEQUAÇÃO NO USO DA PARAMENTAÇÃO CIRÚRGICA
}

\author{
Assessment of adequacy in the use of surgical attire
}

\author{
Evaluación de la aptitud en el uso de paramentación quirúrgica \\ Maria Rafaella Carvalho de Jesus ${ }^{1 *} \oplus$, Michele Gutierrez Melo ${ }^{\oplus} \odot$, Maria Pontes de Aguiar Campos $^{2} \oplus$, \\ Tamara Oliveira Barbosa ${ }^{3} \odot$, Ana Cristina Freire Abud ${ }^{4} \odot$, Daniela de Souza Lordel $0^{5} \odot$
}

RESUMO: Objetivo: Analisar a adequação da paramentação cirúrgica pelos profissionais de saúde que prestam assistência em um centro cirúrgico de um hospital de ensino no Nordeste do Brasil. Método: Estudo transversal, descritivo, quantitativo e de natureza observacional. A amostra foi composta de 100 profissionais de saúde. Resultados: Os itens de maior adequação foram: no momento da colocação do propé e das luvas (100\%), seguido da circulação da roupa privativa e do momento de colocação do gorro (99\%). Obtiveram-se menores taxas de adequação no uso dos óculos (5\%) e quanto ao local de guarda da máscara $(8 \%)$. As inadequações relacionadas à máscara cirúrgica ocorreram pela não troca mediante sujidade ou umidade e em cirurgias com duração maior de duas horas aproximadamente (23\%). Conclusão: Apesar de a maioria (18 dos 20) dos itens estar com boas taxas de adequação, outros apresentaram taxas consideradas medianas e baixas. As categorias profissionais que apresentaram maiores inadequações foram anestesistas (35\%), enfermeiros (27\%) e técnicos de enfermagem (22\%). Esses percentuais necessitam ser melhorados, a fim de oferecer aos pacientes cirúrgicos uma assistência mais segura.

Palavras-chave: Centro cirúrgico. Enfermagem perioperatória. Controle de qualidade. Segurança.

ABSTRACT: Objective: To analyze the adequacy of the surgical attire used by healthcare professionals who work in the surgical center of a teaching hospital in Northeastern Brazil. Method: This is a cross-sectional descriptive quantitative observational study. The sample consisted of 100 healthcare professionals. Results: The items with the best adequacy were: donning disposable shoe covers and gloves (100\%), followed by places where professionals circulate with surgical scrubs and donning the surgical cap (99\%). The use of safety glasses (5\%) and the proper place for keeping the surgical mask (8\%) had lower rates of adequacy. We found surgical mask inadequacies related to professionals who do not change the item when it gets dirty or wet and in surgeries lasting more than approximately two hours (23\%). Conclusion: Although most (18 of the 20) items had high rates of adequacy, others presented regular and low rates. Professional categories with the highest rates of inadequacy were anesthetists (35\%), nurses (27\%), and nursing technicians (22\%). These percentages must be improved to provide safer care for surgical patients.

Keywords: Surgicenters. Perioperative nursing. Quality control. Safety.

RESUMEN: Objetivo: analizar la adecuación del apósito quirúrgico por parte de profesionales de la salud que brindan asistencia en un centro quirúrgico de un hospital universitario en el noreste de Brasil. Método: estudio transversal, descriptivo, cuantitativo y observacional. La muestra estuvo compuesta por 100 profesionales de la salud. Resultados: Los artículos más adecuados fueron: al colocar las polainas y al ponerse un guante (100\%), seguido de la circulación de ropa privada y el momento en que se colocó la gorra (99\%). Se obtuvieron tasas más bajas de adecuación en el uso de gafas (5\%) y en términos de la ubicación de la máscara $(8 \%)$. Las deficiencias relacionadas con la máscara quirúrgica ocurrieron debido al no intercambio

'Graduanda em Enfermagem pela Universidade Federal de Sergipe (UFS) - Aracaju (SE), Brasil.

2Doutora em Ciências pela Faculdade de Medicina de Ribeirão Preto (USP-RP). Docente titular do Departamento de Enfermagem da UFS - Aracaju (SE), Brasil.

${ }^{3}$ Enfermeira pela UFS - Aracaju (SE), Brasil.

4Doutora em Enfermagem pela USP-RP. Professora adjunta do Departamento de Enfermagem da UFS - Aracaju (SE), Brasil.

${ }^{5}$ Enfermeira pela Universidade Federal da Bahia (UFBA); pós-graduada sob forma de Residência em Enfermagem Médico-Cirúrgica pela Escola de Enfermagem da UFBA; mestranda do Núcleo de Pós-

graduação em Enfermagem da UFS - Aracaju (SE), Brasil

*Autora correspondente: raffa.carvalho@hotmail.com

Fonte de financiamento: nenhuma.

Recebido: 12/09/2019 - Aprovado: 19/02/2020

DOI: $10.5327 / Z 1414-4425202000020005$ 
debido a la suciedad o la humedad, y en cirugías que duraron más de dos horas, aproximadamente (23\%). Conclusión: Aunque la mayoría (18 de los 20 ítems) tuvieron buenas tasas de adecuación, otros tuvieron tasas consideradas medianas y bajas. Las categorías profesionales que mostraron las mayores deficiencias fueron anestesistas (35\%), enfermeras (27\%) y técnicos de enfermería (22\%). Es necesario mejorar estos porcentajes para ofrecer a los pacientes quirúrgicos una atención más segura.

Palabras clave: Centros quirúrgicos. Enfermería perioperatoria. Control de calidad. Seguridad.

\section{INTRODUÇÃO}

O centro cirúrgico (CC) é o lugar mais propenso a oferecer riscos, e a presença de eventos adversos durante um procedimento anestésico-cirúrgico está estimada em $37,6 \%{ }^{1}$. Estudo conduzido pelo Ministério da Saúde (MS) afirma que, no Brasil, a infecção do sítio cirúrgico (ISC) apresenta taxa de $11 \%$ em relação ao total dos procedimentos cirúrgicos analisados $^{2-5}$. Com isso, alguns cuidados devem ser realizados pelos profissionais, entre eles, o uso apropriado da paramentação cirúrgica e dos equipamentos de proteção individual (EPI), com a finalidade de a cirurgia ocorrer de forma adequada e segura para o paciente e para os profissionais envolvidos ${ }^{2,6}$. Tais fatos justificam a presente pesquisa.

A paramentação cirúrgica é um conjunto de barreiras contra a invasão de microrganismos nos sítios cirúrgicos e para proteção de exposição dos profissionais a sangue e outros fluidos orgânicos de pacientes. Essas barreiras compõem-se de roupa privativa, gorro/ touca, propé, máscara cirúrgica, óculos cirúrgicos, avental e luva cirúrgica ${ }^{5,7-10}$.

A troca da roupa privativa é indicada sempre que o profissional entrar na área restrita do CC, de acordo com a Association of periOperative Registered Nurses (AORN), e deve ser retirada ao sair (diariamente ou no final de cada turno) ou quando a roupa estiver molhada, suja ou contaminada ${ }^{6,11}$. O uso do gorro é considerado adequado quando cobre totalmente o cabelo, de forma que não existam fios de cabelo expostos, que possam cair no sítio cirúrgico aberto ${ }^{6,10,12}$.

A Associação Brasileira de Enfermeiros de Centro Cirúrgico, Recuperação Anestésica e Centro de Material e Esterilização (SOBECC) $)^{2}$ recomenda o uso de máscara cirúrgica e protetores oculares, indicando que a máscara cirúrgica deve ser substituída após duas horas de uso, quando suja ou úmida, e guardada no bolso do jaleco privativo.

Os sapatos/propés devem estar limpos, sem furos e fechados e seu uso é destinado somente ao ambiente do CC. As luvas cirúrgicas devem ser trocadas quando houver suspeitas de contaminação, se estiverem perfuradas, e a cada 90 a 150 minutos de duração da cirurgia. O avental cirúrgico, ao ser vestido, só poderá ser tocado pela face interna, pois a parte externa anterior é estéril, sendo a parte posterior considerada não estéril ${ }^{2}$.

Ante o exposto, pretende-se responder aos seguintes questionamentos: quais são os elementos da paramentação cirúrgica que apresentam maior adequação de uso em uma instituição de ensino superior? Qual é o percentual de adequação de acordo com a categoria profissional?

Em função da importância da prática de cirurgia segura, este estudo visa contribuir diretamente com a instituição na qual foi realizada a pesquisa por meio do diagnóstico comportamental dos profissionais que laboram no CC e, desse modo, levá-los ao uso mais consciente e adequado da paramentação cirúrgica, além de contribuir com uma assistência multiprofissional mais segura e de maior qualidade aos pacientes.

\section{OBJETIVO}

Analisar o uso adequado da paramentação cirúrgica pelos profissionais de saúde que prestam assistência em um CC de um hospital de ensino no Nordeste do Brasil.

\section{MÉTODO}

Estudo transversal, descritivo, de natureza observacional, com abordagem quantitativa. O enfoque transversal é capaz de visualizar a situação de uma população em um determinado momento. As investigações descritiva e quantitativa têm como objetivo primordial descrever as características de determinada população ou fenômeno ou então estabelecer relação entre as variáveis, utilizando o emprego de técnicas padronizadas de coleta de dados, tais como questionário e observação sistemática ${ }^{13}$.

Oambiente de pesquisa foi um hospital de ensino, localizado no Nordeste do Brasil (Aracaju, Sergipe). O referido hospital presta assistência de média e alta complexidade, é caracterizado como médio porte e conveniado ao Sistema Único de Saúde (SUS). Possui 109 leitos de internação, distribuídos em clínicas médica, cirúrgica e pediátrica, centros cirúrgicos e unidade de terapia intensiva. 
A unidade de observação foi o CC, que possui cinco salas de operação (SO), porém quatro em funcionamento, uma sala de recuperação pós-anestésica (SRPA) com cinco leitos e uma sala de admissão e preparo. Essa unidade realiza, por mês, em média 176 cirurgias eletivas de diversas especialidades.

A população-alvo do estudo foi composta de 192 profissionais de saúde: 6 enfermeiros, 26 técnicos de enfermagem, 34 médicos cirurgiões, 42 anestesiologistas, 9 residentes de anestesia e 75 residentes de cirurgia. Contudo a amostra resultou em 100 profissionais. A casuística desta pesquisa foi não probabilística e intencional.

$\mathrm{O}$ cálculo do tamanho da amostra foi $\mathrm{n} \times$ número de itens, em que $n$ é o número eleito de participantes e número de itens é o número de itens do instrumento de pesquisa. Para se obter um tamanho de amostra ideal para este estudo, elegeu-se cinco participantes por cada item respondido, cujo instrumento foi composto de 20 itens. Assim, a amostra desta pesquisa compreendeu 100 profissionais atuantes no CC, o que corresponde a uma amostra adequada, segundo Mokkink ${ }^{14}$.

Os critérios de inclusão da amostra foram: estar lotado no CC, aceitar participar da pesquisa por meio da assinatura do termo de consentimento livre e esclarecido (TCLE) e estar presente no setor por ocasião da coleta de dados. Os critérios de não inclusão foram: profissional de saúde do CC que trabalha no plantão noturno e que estava gozando férias, licença, folgas ou apresentava faltas no período destinado à coleta de dados.

As entrevistas foram realizadas pelas pesquisadoras, nos turnos matutino e vespertino, de segunda a sexta-feira, no período de junho a julho de 2019. Para tanto, elaborou-se, pelas autoras, um questionário estruturado contendo 20 perguntas fechadas, distribuído entre roupa privativa, gorro, propé, máscara cirúrgica, avental cirúrgico, luva cirúrgica e óculos. No caso dos três últimos itens, a coleta restringiu-se a cirurgiões, residentes de cirurgia e instrumentadores, e o último item incluía os circulantes. O questionário foi elaborado com base nos guidelines da AORN, da SOBECC e da Organização Mundial da Saúde (OMS), bem como em artigos científicos. As variáveis para cada item foram categorizadas em adequada, inadequada e não se aplica. Inicialmente, aplicou-se o teste piloto, e as inconsistências observadas foram corrigidas e reaplicadas.

Realizaram-se duas observações sistemáticas e indiretas para não provocar constrangimentos, desde a chegada do profissional para o uso da paramentação cirúrgica até sua saída do CC. Cada rodada de observação foi realizada por uma pesquisadora, em dias diferentes e por categoria profissional, a fim de conferir fidedignidade aos dados.
Os dados coletados foram compilados em um banco de dados do Microsoft Office Excel (versão 2007) e foi usada a função CONT.SE. A etapa de lançamento dos dados teve dupla digitação, pelas próprias pesquisadoras, para minimizar possibilidades de erros. Em seguida, aplicou-se a análise dos dados pelas frequências absolutas e por percentual simples. Os resultados estão apresentados em quadros, para melhor visualização, os quais foram analisados e discutidos conforme literatura pertinente e atualizada.

O desenvolvimento do estudo atendeu às normas de ética em pesquisas com seres humanos e foi aprovado pelo Comitê de Ética em Pesquisa (CEP), CAAE n ${ }^{\circ}$ 68030317.4.0000.5546, número do parecer: 2.099.033.

\section{RESULTADOS}

Participaram do estudo 100 profissionais, correspondendo a $52,08 \%$ da população, constituídos de 25 médicos cirurgiões, 24 residentes cirurgiões, quatro residentes anestesistas, 15 anestesistas, seis enfermeiros e 26 técnicos de enfermagem, sendo 20 técnicos circulantes e seis técnicos instrumentadores.

A média de idade dos sujeitos da pesquisa foi de 38,4 anos, sendo médicos cirurgiões 44,72 anos; residentes 29,32 anos; anestesistas 42,8 anos; enfermeiros 35,5 anos e técnicos de enfermagem 38,84 anos.

Em relação ao sexo, observou-se percentual semelhante entre o masculino e o feminino, correspondendo a 59 (59\%) e $41(41 \%)$, respectivamente.

Observou-se que houve maior adequação no momento da colocação do propé e na colocação de luvas ( $100 \%$ cada), seguida do local de circulação da roupa privativa e do momento de colocação do gorro (99\%), percentual semelhante ao da colocação do avental e à maneira de passar seu cadarço (96\%). Contrariamente, os itens de maior inadequação foram o uso dos óculos (95\%), local de guarda da máscara cirúrgica $(92 \%)$, modelo de gorro $(64 \%)$ e cobertura do gorro (35\%) (Quadro 1).

Analisando a adequação no uso da paramentação cirúrgica por categoria profissional, constatou-se que médicos cirurgiões, residentes, enfermeiros e técnicos de enfermagem obtiveram $100 \%$ de adequação referente aos itens de locais de circulação de roupa privativa, momento de colocação do gorro, momento de colocação do propé e momento de colocação das luvas. Entre esses itens, a categoria dos anestesistas foi $100 \%$ adequada apenas no momento de colocação do propé (Quadro 2). 
Quadro 1. Uso da paramentação cirúrgica segundo os critérios de adequação e inadequação.

\begin{tabular}{|c|c|c|c|c|c|}
\hline Itens & & Número & Adequado (\%) & Inadequado (\%) & Total $(\%)$ \\
\hline \multirow{2}{*}{ Roupa privativa } & Circulação & 100 & 99 & 1 & 100 \\
\hline & Tamanho & 100 & 87 & 13 & 100 \\
\hline \multirow{3}{*}{ Gorro } & Colocação & 100 & 99 & 1 & 100 \\
\hline & Cobertura & 100 & 65 & 35 & 100 \\
\hline & Modelo & 100 & 36 & 64 & 100 \\
\hline \multirow{2}{*}{ Propé } & Momento de colocação & 100 & 100 & 0 & 100 \\
\hline & Modelo & 100 & 78 & 22 & 100 \\
\hline \multirow{4}{*}{ Máscara cirúrgica } & Cobertura & 100 & 75 & 25 & 100 \\
\hline & Ambiente de uso & 100 & 91 & 9 & 100 \\
\hline & Troca & 100 & 78 & 22 & 100 \\
\hline & Local de guarda & 100 & 8 & 92 & 100 \\
\hline \multirow{5}{*}{ Avental Cirúrgico* } & Colocação & 100 & 96 & 4 & 100 \\
\hline & Troca & 100 & 87 & 13 & 100 \\
\hline & Maneira de passar o cadarço & 100 & 96 & 4 & 100 \\
\hline & Momento de colocação & 100 & 84 & 16 & 100 \\
\hline & Tamanho & 100 & 93 & 7 & 100 \\
\hline \multirow{3}{*}{ Luva Cirúrgica* } & Tamanho & 100 & 94 & 6 & 100 \\
\hline & Troca & 100 & 91 & 9 & 100 \\
\hline & Momento de colocação & 100 & 100 & 0 & 100 \\
\hline Óculos** & Ambiente de uso & 100 & 5 & 95 & 100 \\
\hline
\end{tabular}

*Avental cirúrgico, luva cirúrgica e óculos aplicados aos cirurgiões, instrumentadores e residentes em cirurgia; **óculos incluem os circulantes de salas.

Quadro 2. Uso da paramentação cirúrgica segundo categoria profissional, em relação aos critérios de adequação e inadequação.

\begin{tabular}{|c|c|c|c|c|c|c|c|c|c|c|c|c|c|c|c|c|c|c|c|c|c|}
\hline \multirow{2}{*}{\multicolumn{2}{|c|}{$\frac{\text { Categoria profissional }}{\text { Adequação } n=100(\%)}$}} & \multicolumn{4}{|c|}{ Cirurgião } & \multicolumn{4}{|c|}{ Anestesista } & \multicolumn{4}{|c|}{ Residente } & \multicolumn{4}{|c|}{ Enfermeiro } & \multicolumn{4}{|c|}{ Técnico em enfermagem } \\
\hline & & $\mathbf{n}$ & A & I & $\mathbf{T}$ & $\mathbf{n}$ & A & I & $\mathbf{T}$ & $\mathbf{n}$ & A & I & $\mathbf{T}$ & $\mathbf{n}$ & A & I & $\mathbf{T}$ & $\mathbf{n}$ & A & I & $\mathbf{T}$ \\
\hline \multirow{2}{*}{$\begin{array}{l}\text { Roupa } \\
\text { privativa }\end{array}$} & Circulação & 25 & $100 \%$ & $0 \%$ & $100 \%$ & 15 & $93 \%$ & $7 \%$ & $100 \%$ & 28 & $100 \%$ & $0 \%$ & $100 \%$ & 6 & $100 \%$ & $0 \%$ & $100 \%$ & 26 & $100 \%$ & $0 \%$ & $100 \%$ \\
\hline & Tamanho & 25 & $80 \%$ & $20 \%$ & $100 \%$ & 15 & $93 \%$ & $7 \%$ & $100 \%$ & 28 & $89 \%$ & $11 \%$ & $100 \%$ & 6 & $50 \%$ & $50 \%$ & $100 \%$ & 26 & $96 \%$ & $4 \%$ & $100 \%$ \\
\hline \multirow{3}{*}{ Gorro } & Colocação & 25 & $100 \%$ & $0 \%$ & $100 \%$ & 15 & $93 \%$ & $7 \%$ & $100 \%$ & 28 & $100 \%$ & $0 \%$ & $100 \%$ & 6 & $100 \%$ & $0 \%$ & $100 \%$ & 26 & $100 \%$ & $0 \%$ & $100 \%$ \\
\hline & Cobertura & 25 & $36 \%$ & $64 \%$ & $100 \%$ & 15 & $53 \%$ & $47 \%$ & $100 \%$ & 28 & $68 \%$ & $32 \%$ & $100 \%$ & 6 & $67 \%$ & $33 \%$ & $100 \%$ & 26 & $69 \%$ & $31 \%$ & $100 \%$ \\
\hline & Modelo & 25 & $16 \%$ & $84 \%$ & $100 \%$ & 15 & $20 \%$ & $80 \%$ & $100 \%$ & 28 & $32 \%$ & $68 \%$ & $100 \%$ & 6 & $83 \%$ & $17 \%$ & $100 \%$ & 26 & $58 \%$ & $42 \%$ & $100 \%$ \\
\hline \multirow[t]{2}{*}{ Propé } & $\begin{array}{l}\text { Momento de } \\
\text { colocação }\end{array}$ & 25 & $100 \%$ & $0 \%$ & $100 \%$ & 15 & $100 \%$ & $0 \%$ & $100 \%$ & 28 & $100 \%$ & $0 \%$ & $100 \%$ & 6 & $100 \%$ & $0 \%$ & $100 \%$ & 26 & $100 \%$ & $0 \%$ & $100 \%$ \\
\hline & Modelo & 25 & $92 \%$ & $8 \%$ & $100 \%$ & 15 & $87 \%$ & $13 \%$ & $100 \%$ & 28 & $82 \%$ & $18 \%$ & $100 \%$ & 6 & $67 \%$ & $33 \%$ & $100 \%$ & 26 & $58 \%$ & $42 \%$ & $100 \%$ \\
\hline \multirow{4}{*}{$\begin{array}{l}\text { Máscara } \\
\text { cirúrgica }\end{array}$} & Cobertura & 25 & $92 \%$ & $8 \%$ & $100 \%$ & 15 & $33 \%$ & $67 \%$ & $100 \%$ & 28 & $82 \%$ & $18 \%$ & $100 \%$ & 6 & $50 \%$ & $50 \%$ & $100 \%$ & 26 & $81 \%$ & $19 \%$ & $100 \%$ \\
\hline & $\begin{array}{l}\text { Ambiente de } \\
\text { uso }\end{array}$ & 25 & $92 \%$ & $8 \%$ & $100 \%$ & 15 & $73 \%$ & $27 \%$ & $100 \%$ & 28 & $96 \%$ & $4 \%$ & $100 \%$ & 6 & $83 \%$ & $17 \%$ & $100 \%$ & 26 & $96 \%$ & $4 \%$ & $100 \%$ \\
\hline & Troca & 25 & $80 \%$ & $20 \%$ & $100 \%$ & 15 & $73 \%$ & $27 \%$ & $100 \%$ & 28 & $79 \%$ & $21 \%$ & $100 \%$ & 6 & $100 \%$ & $0 \%$ & $100 \%$ & 26 & $73 \%$ & $27 \%$ & $100 \%$ \\
\hline & $\begin{array}{l}\text { Local de } \\
\text { guarda }\end{array}$ & 25 & $92 \%$ & $8 \%$ & $100 \%$ & 15 & $0 \%$ & $100 \%$ & $100 \%$ & 28 & $18 \%$ & $82 \%$ & $100 \%$ & 6 & $0 \%$ & $100 \%$ & $100 \%$ & 26 & $4 \%$ & $96 \%$ & $100 \%$ \\
\hline \multirow{5}{*}{$\begin{array}{l}\text { Avental } \\
\text { cirúrgico }\end{array}$} & Colocação & 25 & $96 \%$ & $4 \%$ & $100 \%$ & NA & NA & NA & NA & 24 & $96 \% *$ & $4 \% *$ & $100 \%$ & NA & NA & NA & NA & 6 & $100 \%$ ** & $0 \%$ ** & $100 \%$ \\
\hline & Troca & 25 & $84 \%$ & $16 \%$ & $100 \%$ & NA & NA & NA & NA & 24 & $92 \% *$ & $8 \% *$ & $100 \%$ & NA & NA & NA & NA & 6 & $83 \%$ ** & $17 \%$ ** & $100 \%$ \\
\hline & $\begin{array}{c}\text { Maneira de } \\
\text { passar o } \\
\text { cadarço }\end{array}$ & 25 & $96 \%$ & $4 \%$ & $100 \%$ & NA & NA & NA & NA & 24 & $100 \% *$ & $0 \% *$ & $100 \%$ & NA & NA & NA & NA & 6 & $83 \% \%^{* *}$ & $17 \%$ ** & $100 \%$ \\
\hline & $\begin{array}{l}\text { Momento de } \\
\text { colocação }\end{array}$ & 25 & $96 \%$ & $4 \%$ & $100 \%$ & NA & NA & NA & NA & 24 & $71 \% *$ & $29 \%$ & $100 \%$ & NA & NA & NA & NA & 6 & $83 \%$ ** & $17 \%^{* \star}$ & $100 \%$ \\
\hline & Tamanho & 25 & $96 \%$ & $4 \%$ & $100 \%$ & NA & NA & NA & NA & 24 & $96 \% *$ & $4 \%^{*}$ & $100 \%$ & NA & NA & NA & NA & 6 & $67 \%$ ** & $33 \% \%^{* *}$ & $100 \%$ \\
\hline \multirow{3}{*}{$\begin{array}{l}\text { Luva } \\
\text { cirúrgica }\end{array}$} & Tamanho & 25 & $100 \%$ & $0 \%$ & $100 \%$ & NA & NA & NA & NA & 24 & $87 \% *$ & $13 \%$ & $100 \%$ & NA & NA & NA & NA & 6 & $100 \%$ ** & $0 \%$ ** & $100 \%$ \\
\hline & Troca & 25 & $88 \%$ & $12 \%$ & $100 \%$ & NA & NA & NA & NA & 24 & $92 \% *$ & $8 \% *$ & $100 \%$ & NA & NA & NA & NA & 6 & $100 \%$ ** & $0 \%$ ** & $100 \%$ \\
\hline & $\begin{array}{l}\text { Momento de } \\
\text { colocação }\end{array}$ & 25 & $100 \%$ & $0 \%$ & $100 \%$ & NA & NA & NA & NA & 24 & $100 \% *$ & $0 \% *$ & $100 \%$ & NA & NA & NA & NA & 6 & $100 \%$ ** & $0 \%$ ** & $100 \%$ \\
\hline Óculos & $\begin{array}{l}\text { Ambiente de } \\
\text { uso }\end{array}$ & 25 & $8 \%$ & $92 \%$ & $100 \%$ & NA & NA & NA & NA & 24 & $8 \% *$ & $92 \% *$ & $100 \%$ & NA & NA & NA & NA & 26 & $0 \%$ & $100 \%$ & $100 \%$ \\
\hline
\end{tabular}

*As taxas referem-se aos residentes cirurgiões; **os números referem-se aos instrumentadores; A: adequado; I: inadequado; NA: não se aplica; n: número absoluto; T: total. 
As maiores taxas de inadequação foram observadas na não utilização dos óculos, por todas as categorias, e no não uso do gorro cobrindo todo o couro cabeludo, com predomínio das categorias de cirurgiões e anestesistas (64 e $47 \%$, respectivamente). Chama atenção o achado de que os enfermeiros e os anestesistas apresentaram $100 \%$ de inadequação relacionada ao local de guarda das máscaras cirúrgicas (Quadro 2).

No que se refere à adequação geral no uso da paramentação cirúrgica, relacionada com a categoria profissional, apresentaram maiores taxas de inadequação, pela ordem, anestesistas (35\%), enfermeiros (27\%) e técnicos de enfermagem (22\%) (Quadro 2).

Para as variáveis propé, máscara, avental e luva cirúrgica, foram analisados seus itens para maior entendimento da problemática em questão (Quadro 3).

Observou-se que o propé mais inadequado foi aquele com modelo de furo, com uso predominante pelas categorias: instrumentador $(50 \%)$, circulante de sala (40\%) e enfermeiros (33\%) (Quadro 3).

Quadro 3. Itens da paramentação cirúrgica tipificando as adequações e inadequações, segundo a categoria profissional.

\begin{tabular}{|c|c|c|c|c|c|c|c|c|c|c|c|c|c|c|c|c|c|}
\hline \multirow{3}{*}{\multicolumn{4}{|c|}{ Itens analisados }} & \multicolumn{14}{|c|}{ Categoria profissional } \\
\hline & & & & \multicolumn{2}{|c|}{$\begin{array}{l}\text { Médico } \\
\text { Cirurgião }\end{array}$} & \multicolumn{2}{|c|}{ Anestesista } & \multicolumn{2}{|c|}{$\begin{array}{l}\text { Residente } \\
\text { cirurgiáo }\end{array}$} & \multicolumn{2}{|c|}{$\begin{array}{l}\text { Residente } \\
\text { anestesia }\end{array}$} & \multicolumn{2}{|c|}{ Enfermeiro } & \multicolumn{2}{|c|}{ Circulante } & \multicolumn{2}{|c|}{ Instrumentador } \\
\hline & & & & $\mathbf{n}$ & $x(\%)$ & n & $x(\%)$ & $\mathbf{n}$ & $x(\%)$ & $\mathbf{n}$ & $x(\%)$ & $\mathbf{n}$ & x $(\%)$ & $\mathbf{n}$ & $x(\%)$ & $\mathbf{n}$ & x (\%) \\
\hline \multirow{4}{*}{ Propé } & \multirow{4}{*}{ Modelo } & \multirow{2}{*}{ Adequado } & Propé & 25 & 80 & 15 & 73 & 24 & 75 & 4 & 50 & 6 & 50 & 20 & 45 & 6 & 33 \\
\hline & & & Calçado sem furo & 25 & 12 & 15 & 13 & 24 & 8 & 4 & 25 & 6 & 17 & 20 & 15 & 6 & 17 \\
\hline & & \multirow{2}{*}{ Inadequado } & Calçado com furo & 25 & 8 & 15 & 13 & 24 & 17 & 4 & 25 & 6 & 33 & 20 & 40 & 6 & 50 \\
\hline & & & Não utiliza & 25 & 0 & 15 & 0 & 24 & 0 & 4 & 0 & 6 & 0 & 20 & 0 & 6 & 0 \\
\hline \multirow{8}{*}{$\begin{array}{l}\text { Máscara } \\
\text { Cirúrgica }\end{array}$} & \multirow{4}{*}{ Cobertura } & Adequado & $\begin{array}{l}\text { Cobre total } \\
\text { cirurgia total }\end{array}$ & 25 & 92 & 15 & 33 & 24 & 87 & 4 & 50 & 6 & 50 & 20 & 75 & 6 & 100 \\
\hline & & \multirow{3}{*}{ Inadequado } & $\begin{array}{c}\text { Cobre parcial início } \\
\text { ou fim }\end{array}$ & 25 & 4 & 15 & 27 & 24 & 0 & 4 & 0 & 6 & 17 & 20 & 5 & 6 & 0 \\
\hline & & & $\begin{array}{l}\text { Cobre parcial } \\
\text { durante toda } \\
\text { cirurgia }\end{array}$ & 25 & 4 & 15 & 33 & 24 & 13 & 4 & 50 & 6 & 33 & 20 & 20 & 6 & 0 \\
\hline & & & Não utiliza & 25 & 0 & 15 & 7 & 24 & 0 & 4 & 0 & 6 & 0 & 20 & 0 & 6 & 0 \\
\hline & \multirow{4}{*}{ Troca } & \multirow{3}{*}{ Adequado } & $\begin{array}{c}\text { Troca em duas } \\
\text { horas }\end{array}$ & 25 & 0 & 15 & 0 & 24 & 0 & 4 & 25 & 6 & 0 & 20 & 0 & 6 & 0 \\
\hline & & & $\begin{array}{l}\text { Troca quando } \\
\text { sujo/úmido }\end{array}$ & 25 & 0 & 15 & 0 & 24 & 0 & 4 & 0 & 6 & 0 & 20 & 0 & 6 & 0 \\
\hline & & & NA & 25 & 80 & 15 & 80 & 24 & 75 & 4 & 75 & 6 & 100 & 20 & 80 & 6 & 67 \\
\hline & & Inadequado & $\begin{array}{l}\text { Não troca nessas } \\
\text { situações }\end{array}$ & 25 & 20 & 15 & 20 & 24 & 25 & 4 & 0 & 6 & 0 & 20 & 20 & 6 & 33 \\
\hline \multirow{5}{*}{$\begin{array}{l}\text { Avental } \\
\text { cirúrgico }\end{array}$} & \multirow{5}{*}{ Troca } & \multirow{3}{*}{ Adequado } & $\begin{array}{l}\text { Troca quando } \\
\text { sujo/úmido }\end{array}$ & 25 & 4 & NA & NA & 24 & 0 & NA & NA & NA & NA & NA & NA & 6 & 0 \\
\hline & & & $\begin{array}{l}\text { Troca quando } \\
\text { contamina }\end{array}$ & 25 & 0 & NA & NA & 24 & 4 & NA & NA & NA & NA & NA & NA & 6 & 0 \\
\hline & & & NA & 25 & 80 & NA & NA & 24 & 88 & NA & NA & NA & NA & NA & NA & 6 & 83 \\
\hline & & \multirow{2}{*}{ Inadequado } & $\begin{array}{l}\text { Não troca quando } \\
\text { contamina }\end{array}$ & 25 & 0 & NA & NA & 24 & 4 & NA & NA & NA & NA & NA & NA & 6 & 17 \\
\hline & & & $\begin{array}{l}\text { Não troca quando } \\
\text { sujo/úmido }\end{array}$ & 25 & 16 & NA & NA & 24 & 4 & NA & NA & NA & NA & NA & NA & 6 & 0 \\
\hline \multirow{5}{*}{$\begin{array}{l}\text { Luva } \\
\text { cirúrgica }\end{array}$} & \multirow{5}{*}{ Troca } & \multirow{3}{*}{ Adequado } & $\begin{array}{c}\text { Troca após duas } \\
\text { horas }\end{array}$ & 25 & 4 & NA & NA & 24 & 12 & NA & NA & NA & NA & NA & NA & 6 & 0 \\
\hline & & & $\begin{array}{l}\text { Troca quando } \\
\text { perfura }\end{array}$ & 25 & 8 & NA & NA & 24 & 0 & NA & NA & NA & NA & NA & NA & 6 & 33 \\
\hline & & & NA & 25 & 76 & NA & NA & 24 & 76 & NA & NA & NA & NA & NA & NA & 6 & 0 \\
\hline & & \multirow{2}{*}{ Inadequado } & $\begin{array}{c}\text { Não troca em } \mathrm{C}>2 \\
\text { horas }\end{array}$ & 25 & 12 & NA & NA & 24 & 12 & NA & NA & NA & NA & NA & NA & 6 & 67 \\
\hline & & & $\begin{array}{c}\text { Não troca quando } \\
\text { perfura }\end{array}$ & 25 & 0 & NA & NA & 24 & 0 & NA & NA & NA & NA & NA & NA & 6 & 0 \\
\hline
\end{tabular}

${ }^{*} \mathrm{C}$ : cirurgia;**NA: não se aplica; n: número absoluto; $x$ : variável. 
Com relação ao uso da máscara cirúrgica, as maiores taxas de inadequação foram identificadas nos anestesistas (67\%), em que se observou cobertura parcial no início ou no final da cirurgia (27\%), cobertura parcial durante todo o procedimento cirúrgico (33\%) e não uso da máscara (7\%), seguidos pelos enfermeiros $(50 \%)$. Ainda em relação ao uso da máscara cirúrgica, quanto à periodicidade de troca, as maiores taxas de inadequação foram praticadas pelo instrumentador (33\%), pelos residentes de cirurgia (25\%) e pelos cirurgiões (20\%) (Quadro 3).

Quanto ao uso do avental cirúrgico, no que diz respeito à troca preconizada, verificou-se que em 17,16 e $8 \%$ das cirurgias não houve troca pelos instrumentadores, cirurgiões e residentes de cirurgia, respectivamente (Quadro 3).

Em relação à troca de luvas cirúrgicas, que deve ser realizada após duas horas da cirurgia e em caso de perfuração, os maiores percentuais de inadequação apresentam-se nas seguintes categorias: $67 \%$ pelo instrumentador, $12 \%$ para os residentes de cirurgia e $12 \%$ para os cirurgiões (Quadro 3).

\section{DISCUSSÃO}

O uso da paramentação cirúrgica é importante dada sua função de formar uma barreira microbiológica contra a penetração de microrganismos diretamente no sítio cirúrgico do paciente, que podem ser procedentes dele mesmo, dos próprios profissionais envolvidos na cirurgia, de equipamentos, entre outros ${ }^{9,10,15}$.

Considera-se adequado o tamanho da roupa privativa que garante cobertura completa da cintura e dos membros inferiores, evitando a área exposta ${ }^{10,15,16}$. No presente estudo, verificou-se que $87 \%$ das observações estavam adequadas (Quadro 1). Esse achado é relevante, pois o uso de roupas privativas é uma das ferramentas mais ativas na preservação à saúde e na inteireza física do colaborador, ajudando na prevenção de contaminações, de modo que sua utilização incorreta pode afetar esse processo ${ }^{6}$.

Em relação ao gorro, a literatura científica recomenda que deve cobrir todo o couro cabeludo, com elástico em toda a sua abertura, podendo ser de tecido de algodão reprocessável até Spunbonbed, Meltblown, Spunbonbed (SMS), ou seja, tecido não tecido de uso único ${ }^{2,12,15}$. Foi observado que pouco mais da metade $(65 \%)$ dos profissionais cobria adequadamente a cabeça. Taxas semelhantes de inadequação foram constatadas em relação ao modelo de gorro utilizado (Quadro 1). Resultado diferente foi observado em estudo ${ }^{7}$ realizado em um hospital universitário de Belo Horizonte (Minas Gerais), onde o uso do gorro foi inadequado em, aproximadamente, nove vezes menos em relação a este estudo?.

A máscara deve cobrir completamente o nariz, a boca e as regiões laterais e ser transportada no bolso da roupa privativa. Caso seja colocada no pescoço, essa atitude é inadequada pela exposição antecipada de colonização ${ }^{6,12}$. O seu uso é obrigatório ao entrar na sala operatória e no lavabo ${ }^{2,15}$. Sua utilização, quanto aos locais de uso obrigatório, foi adequada e ocorreu, neste estudo, em 91\% dos profissionais (Quadro 1), corroborando o estudo que avaliou a adesão às medidas para prevenção de ISC em um CC de um hospital público ${ }^{7}$. A boa adesão ao uso da máscara talvez se deva ao fato de que não existem controvérsias quanto à sua eficácia no controle das infecções.

O avental cirúrgico deve permitir cobertura completa do tronco, a partir do pescoço, membros superiores até os punhos e membros inferiores até os joelhos, com livre movimentação. Deve ser utilizado por cirurgiões, residentes de cirurgia e instrumentadores, logo após a degermação e secagem das mãos, devendo ficar até o término da cirurgia, sendo necessária a troca em casos de contaminação ou quando úmido ou sujo ${ }^{10,15}$. Nesses aspectos, a maioria dos profissionais observados usou adequadamente, atingindo quase a totalidade das observações quanto ao momento da colocação, à maneira de passar o cadarço, ao tamanho e à troca (Quadro 1). Esses resultados aproximam-se do observado em estudo ${ }^{7}$ no qual foi obtida a integralidade da equipe cirúrgica utilizando-o de forma adequada.

As luvas cirúrgicas devem ser de boa qualidade, no que diz respeito à flexibilidade, à impermeabilidade e à resistência ao tempo e aos movimentos cirúrgicos. Para tanto, devem ser de látex, esterilizadas, descartáveis e substituídas sempre que forem perfuradas e em cirurgias de longa duração $0^{6,10,15}$. Esse item obteve as maiores taxas de adequação em relação à troca e à colocação no momento certo, aproximando-se do todo das observações (Quadro 1). Esses resultados concordam com estudo em que foi obtida adequação de $100 \%$ no uso correto das luvas ${ }^{7}$. Tal achado é de extrema relevância, pois, como referem autores ${ }^{12,17}$, a utilização de luvas cirúrgicas estéreis é uma medida fundamental de prevenção da ISC, servindo como barreira física para os microrganismos presentes nas mãos dos profissionais de saúde, no ambiente e nos pacientes. Além disso, a falha na integridade viabiliza a transferência dos microrganismos, aumentando duas vezes o risco de ISC.

O uso dos óculos é indicado para proteção ocupacional, isto porque impedem contato direto dos olhos com exsudatos do paciente ${ }^{10,15,18}$. Contudo a adesão tem se deparado com resistência, provavelmente em razão da diminuição da acuidade visual e da perda da transparência das lentes pela respiração 
para dentro do visor ${ }^{10,15}$. Para a seleção dos óculos, deve ser considerada a presença de viseiras largas de acrílico ou vidro e protetores da face contra fluidos ${ }^{15}$. É provável que o não fornecimento desse EPI, conforme determina a legislação vigente, pode justificar ter sido esse item o que obteve a mais baixa taxa de adesão (Quadro 1) entre todos os itens da paramentação cirúrgica, corroborando o estudo de Freiberger et al. ${ }^{18}$.

Sapatos privativos e propés formam barreiras contra organismos patogênicos presentes nos sapatos. Sua relevância no controle da ISC está na probabilidade da contaminação pelas mãos quando tocam os sapatos ${ }^{15}$. Atualmente, há controvérsia quanto à exigência do uso pelo fato de que sua utilização não previne a contaminação ambiental e pode levar microrganismos até às mãos dos funcionários quando eles tocam seus pés para retirar os sapatos e não as lavam imediatamente ${ }^{12,19}$. Dessa forma, segundo alguns autores ${ }^{9,12}$, não deve ser considerado como uma barreira de proteção ambiental, já que sua utilização previne apenas que os sapatos dos profissionais sejam sujos por sangue e outros líquidos corpóreos.

Constatou-se, nesse estudo, que esse EPI apresentou a maior taxa de adequação (100\%) para todas as categorias (Quadro 1). Chama atenção o fato de que o EPI de maior adequação foi exatamente aquele que não tem evidência de sua eficácia para a prevenção das infecções. Resultados diferentes foram obtidos em estudo com menores taxas de utilização ${ }^{7}$, provavelmente porque os profissionais do citado estudo estavam conscientes da menor importância dos propés pelas controvérsias existentes em relação à sua eficácia para prevenção das infecções cirúrgicas.

Analisando a adequação/inadequação da paramentação cirúrgica segundo a categoria profissional, obtiveram-se as maiores taxas de inadequação no uso dos óculos e da máscara cirúrgica. Relacionado ao primeiro, foram observadas taxas de inadequação em cirurgiões (92\%), residentes de cirurgia (92\%) e instrumentadores (100\%) (Quadro 1). Embora o uso dos óculos seja preconizado do início ao término da cirurgia e eles devam ser utilizados pelas categorias profissionais cirurgiões, residentes de cirurgia, instrumentadores e, em alguns momentos, por circulantes e anestesistas quando houver risco de respingos, o que chama atenção, no resultado deste estudo, foi o fato desse item ter a maior porcentagem de inadequação, quase a totalidade, em todas as categorias observadas ${ }^{15}$. Quando comparado com outros estudos ${ }^{7,18,20}$, verificam-se resultados similares, o que comprova as dificuldades dos profissionais da equipe cirúrgica de aderirem ao uso dos óculos de proteção.

O segundo EPI que obteve maior taxa de inadequação por categoria profissional foi a máscara cirúrgica, em relação à maneira adequada de utilizá-la. Constatou-se que as categorias anestesista (67\%) e enfermeiro (50\%) obtiveram maiores taxas de inadequação das situações observadas (Quadro 1). Esse dado é preocupante, pois, segundo a literatura, o uso correto da máscara cirúrgica é uma atitude eficaz, comprovada há décadas, no controle das infecções cirúrgicas. Resultado diferente foi obtido em estudos ${ }^{7,12}$ nos quais a adesão à utilização correta foi bem maior, quando comparada com esta pesquisa.

Destacam-se como adequados a circulação com a roupa privativa, o tamanho e o momento da colocação da luva cirúrgica pelos cirurgiões, residentes e técnicos de enfermagem. Considera-se significante esse dado, pois, conforme cita a literatura, a colocação deve ser próxima do momento cirúrgico e ocorrer após a colocação do avental, desse modo se diminui a exposição aos microrganismos ${ }^{21}$.

Discutindo a tipificação dos principais itens da paramentação (luvas cirúrgicas e máscaras), as corretas trocas de luvas ocorreram majoritariamente nos casos de perfuração e em cirurgias com duração acima de duas horas, cujas trocas foram efetuadas pelo médico cirurgião e residente de cirurgia (ambos $88 \%$ ) e pelo instrumentador (33\%) (Quadro 3). Ocorrência divergente foi verificada em outro estudo, com maior percentual de trocas pelo médico, seguindo em percentuais iguais o auxiliar/assistente e o instrumentador ${ }^{7}$. Ressalta-se um estudo ${ }^{17}$ que aponta que a qualidade do produto deve ser avaliada, pois testes realizados com luvas cirúrgicas não apresentaram risco de perfurações.

Referente à exigência de troca das máscaras, em cirurgias com duração acima de duas horas, quando úmida e na presença de exsudatos, elas não ocorreram em $33 \%$ das observações para os instrumentadores, $25 \%$ para residentes de cirurgia e 20\% em relação aos médicos cirurgiões (Quadro 3). Embora tenham sido observadas taxas pequenas de inadequação, mesmo assim são significativas, dada sua importância como barreiras microbiológicas².

Enfim, a utilização de EPI e sua baixa anuência pelos profissionais de saúde em procedimentos invasivos ainda representa um comportamento que influencia de forma direta ou indireta na segurança do profissional e, sobretudo, do paciente, colocando-os em zona de perigo ${ }^{20}$.

Nesse sentido, entre os papéis que os profissionais atuantes na cirurgia possuem na prevenção dos fatores relacionados a esse procedimento, estudos incluem a adequada paramentação ${ }^{7}$ e a importância de seu uso como meio para reduzir as taxas de infecção ${ }^{12}$. Desse modo, o enfermeiro, na equipe, deve ter papel de destaque, como um agente relevante no estabelecimento de uma assistência segura, orientando e supervisionando o uso da paramentação cirúrgica. 
As limitações deste estudo estiveram relacionadas àquelas tradicionalmente envolvidas em pesquisas de natureza observacional, nesse caso específico, podemos citar: tempo de acompanhamento dos procedimentos cirúrgicos, uma vez que em algumas cirurgias esse tempo foi menor que duas horas, o cancelamento de cirurgias e a escassez de recursos materiais.

\section{CONCLUSÃO}

Após análise dos dados, pôde-se concluir que a maioria, 18 do total de 20 , correspondendo a $90 \%$, dos itens da paramentação cirúrgica apresentou percentuais de adequação, que variaram de 5 a $100 \%$. Destaca-se a colocação de luva e o momento de colocação do propé, com 100\% de adequação, e, com menores taxas, o uso de óculos (5\%) e o local de guarda da máscara cirúrgica ( $8 \%$ ).

As categorias profissionais que apresentaram maiores taxas de inadequações foram os anestesistas (35\%), enfermeiros (27\%) e técnicos de enfermagem (22\%).

Conclui-se ainda que, apesar de a maioria dos itens observados estar com percentuais igual e acima de $75 \%$ de adequação, outros apresentaram percentuais considerados muito baixos (5 e 8\%). Desse modo, é urgente que as taxas sejam melhoradas, a fim de oferecer aos pacientes cirúrgicos uma assistência cada vez mais segura.

\section{REFERÊNCIAS}

1. Manrique BT, Soler LM, Bonmati AN, Montesinos MJL, Roche FP. Segurança do paciente no centro cirúrgico e qualidade documental relacionada à infecção cirúrgica e à hospitalização. Rev Acta Paul Enferm. 2015;28(4):355-60. https://doi.org/10.1590/1982-0194201500060

2. Associação Brasileira de Enfermeiros de Centro Cirúrgico, Recuperação Anestésica e Centro de Material e Esterilização (SOBECC). Diretrizes de práticas em enfermagem cirúrgica e processamento de produtos para a saúde. 7a ed. Barueri: Manole; 2017.

3. Carvalho VM, Moura MEB, Batista OMA, Cruz MP, Sousa MAS, Andrade DFR. Conhecimento dos profissionais de enfermagem sobre fatores de risco relacionados à infecção de sítio cirúrgico. Rev Interd [Internet]. 2015 [acessado em 26 jul. 2019];8(3):1-11. Disponível em: https://revistainterdisciplinar.uninovafapi.edu.br/index.php/revinter/ article/view/596

4. Moraes ACFG. Dificuldades no combate de infecções em centro cirúrgico da rede pública - uma revisão de literatura. Rev Pesq Cuid Fundam Online [Internet]. 2011 [acessado em 4 ago. 2019];3(2):1889-93. Disponível em: http://www.seer.unirio.br/index.php/cuidadofundamental/article/ view/1245/pdf_389

5. Cataneo C, Silveira CA, Simpionato E, Camargo FC, Queiroz FA, Cagnin MC, et al. $O$ preparo da equipe cirúrgica: aspecto relevante no controle da contaminação ambiental. Rev Latino-am Enfermagem. 2004;12(2):283-6. https://doi.org/10.1590/S0104-11692004000200021

6. Madeira MZA, Santana RAP, Santos AMR, Moura ECC. Prevenção de infecção hospitalar pela equipe cirúrgica em um hospital de ensino. Rev SOBECC. 2012;17(1):35-44.

7. Oliveira AC, Gama CS. Avaliação da adesão às medidas para a prevenção de infecções do sítio cirúrgico pela equipe cirúrgica. Rev Esc Enferm USP. 2015;49(5):767-74. https://doi.org/10.1590/ S0080-623420150000500009

8. World Health Organization (WHO). Global guidelines on the prevention surgical site infection [Internet]. Suíça: WHO; 2016 [acessado em 27 maio 2019]. Disponível em: https://www.who.int/gpsc/ ssi-prevention-guidelines/en/
9. Barreto RASS, Rocha-Vilefort LO, Silva ACS, Prado-Palos MA, Barbosa MA, Borges VPFN. Processo de limpeza da sala operatória: riscos à saúde do usuário e do trabalhador. Rev Eletron Enferm [Internet]. 2011 [acessado em 4 ago. 2019];13(2):269-75. Disponível em: https://www. fen.ufg.br/revista/v13/n2/v13n2a13.htm. http://dx.doi.org/10.5216/ ree.v13i2.9191

10. Duarte IGL, Leite MD. Paramentação cirúrgica: artigo de revisão. Rev Médica de Minas Gerais [Internet]. 2013 [acessado em 4 ago. 2019];23(3):343-6. Disponível em: http://rmmg.org/artigo/detalhes/220. http://www.dx.doi.org/10.5935/2238-3182.20130054

11. Mchugh SM, Corrigan MA, Hill ADK, Humphreys H. Surgical attire, practices and their perception in the prevention of surgical site infection. Surgeon [Internet]. 2014 [acessado em 2 jun. 2019];12(1):47-52. Disponível em: https://www.ncbi.nlm.nih.gov/pubmed/24268928. https://doi.org/10.1016/j.surge.2013.10.006

12. Sevilha HA, Paiva LSJ, Poveda VB. Análise das variáveis ambientais em salas cirúrgicas: fontes de contaminação. Rev SOBECC. 2014;19(3):1238. http://dx.doi.org/10.4322/sobecc.2014.019

13. Fernandes AM, Bruchêz A, D’Ávila AAF, Castilhos NC, Olea PM. Metodologia de pesquisa de dissertações sobre inovação: análise bibliométrica. Desafio Online [Internet]. 2018 [acessado em 25 maio 2019];6(1):141-59. Disponivel em: http://www.spell.org.br/ documentos/ver/49112

14. Mokkink LB. Cosmin risk of bias checklist. University Medical Center: Amsterdam Public Health Research Institute [Internet]. The Netherlands, 2018 [acessado em 2 ago. 2019]. Disponível em: https:// www.cosmin.nl/wp-content/uploads/COSMIN-RoB-checklist-V20 -v17_rev3.pdf

15. Tristão GDS, Moura IBP. 0 uso de equipamentos de proteção individual (EPI) e técnicas antissépticas em centro cirúrgico [trabalho de conclusão de curso] [Internet]. Brasília: Universidade Católica de Brasília; 2015 [acessado em 22 jul. 2019]. Disponível em: https://repositorio.ucb.br/jspui/bitstream/123456789/ 10420/1/GiovannadaSilvaTrist\%C3\%A3oelallyBrendaPereiraMoura TCCGraduacao2015.pdf 
16. Braswell ML, Spruce L. Implementing AORN recommended practices for surgical attire. AORN Journal [Internet]. 2012 [acessado em 5 jul. 2019];95(1):122-40. Disponível em: https://aornjournal. onlinelibrary.wiley.com/doi/abs/10.1016/j.aorn.2011.10.017. https:// doi.org/10.1016/j.aorn.2011.10.017

17. Bezerra WR, Bezerra ALQ, Paranaguá TB, Bernardes MJC, Teixeira CC Ocorrência de incidentes em um centro cirúrgico: estudo documental. Rev Eletr Enf [Internet]. 2015 [acessado em 27 maio 2019];17(4). Disponivel em: https://revistas.ufg.br/fen/article/view/33339. https:// doi.org/10.5216/ree.v17i4.33339

18. Freiberger MF, Correia MBR, Pinto EAM, Ferreira EJ. Adesão ao uso dos óculos de proteção individual pelos profissionais de saúde em unidade de centro cirúrgico. Rev Cie Fac Edu Mei Amb [Internet]. 2011 [acessado em 4 ago. 2019];2(2):70-9. Disponível em: http://www. faema.edu.br/revistas/index.php/Revista-FAEMA/article/view/95. https://doi.org/10.31072/rcf.v2i2.95
19. Bardaquim VA, Rodrigues JSM, Ribeiro AA, Silva ALNV, Sousa CP. Microbiota aérea em centro cirúrgico: contribuições da enfermagem no controle de infecção hospitalar. J Health Sci Inst [Internet]. 2012 [acessado em 4 ago. 2019];30(1):48-52. Disponível em: https://www. unip.br/presencial/comunicacao/publicacoes/ics/edicoes/2012/01_ jan-mar/V30_n1_2011_p48-52.pdf

20. Stanganelli NC, Ribeiro RP, Claudio CV, Martins JT, Ribeiro PHVR, Ribeiro BGA. A utilização de equipamentos de proteção individual entre trabalhadores de enfermagem de um hospital público. Rev Cogitare Enferm [Internet]. 2015 [acessado em 24 maio 2019];20(2):345-51. Disponível em: https://revistas.ufpr.br/cogitare/article/view/40118. http://dx.doi.org/10.5380/ce.v20i2.40118

21. PazMSO, Lacerda RA, Monteiro CEC, Conceição VP. Paramentação cirúrgica: avaliação de sua adequação para a prevenção de riscos biológicos em cirurgias. Parte I: a utilização durante as cirurgias. Rev Esc Enferm USP. 2000;34(1):108-17. https://doi.org/10.1590/S0080-62342000000100014 